\title{
Research on DEM Modification and Production of Three-dimensional Images in the Intersection Region of Seawater and Land
}

\author{
Chen Lin, Zhou Yifu, Liu Jianfeng \\ Yellow River Conservancy Technical Institute \\ Kaifeng, China \\ e-mail: cl7188@163.com
}

\begin{abstract}
The phenomena often appears that sea surface is above land, island is under sea surface and distortion of building inter-island in conflux area of water and land when creating three-dimensional image using fusing DEM and image. The paper introduced a fast method of modifying DEM and creating three-dimensional image in the batch by using PCI software. This method was validated by using SPOT5 and DEM in the Zhoushan area. The results showed that PCI could accurately mask the target objects and modify DEM in the batch; the result could be showed in three-dimensional mode by setting many parameters and meet different requirements; the results could fly by setting given route; the file was organized in a systemic mode, which will facilitate management.
\end{abstract}

Keywords-PCI; DEM; 3D image

\section{INTRODUCTION}

DEM is a digital representation on the attribute information of the terrain ${ }^{[1]}$. We can fit a Curved surface product of three-dimensional perspective analysis indivisibility, calculate slope and aspect, Etc on by means of DEM. Especially product 3D RS image among the applications ${ }^{[2,3]}$.

The $3 \mathrm{D}$ is that the image is showed in the threedimensional aspects ${ }^{[4]}$. We can Virtual Reality by way of DEM and the 3D RS image. We can know the general topography and its detailed features ${ }^{[5]}$. It is noted that We need modify the DEM before producing the 3D RS image. It can be realized easily by means of all kinds of RS image processing if the number needed to be modified is little ${ }^{[6]}$. But it is a good idea by means of PCI if the number needed to be modified is lot. This article introduces the method which has been used in the district of ZhouSan.

\section{OUTLINING THE MAIN FEATURES OF THE SOFTWEAR PCI}

PCI Geomatica is developed by PCI company of Cadana, which has the functions of processing RS image 、 photogrammetry 、 GIS spatial analysis 、 professional graphics 、 powerful file management features 、 secondary visual development environment. It can be used in multiple sectors.

\section{A. Image masking by ImageWorks}

First, open the image and Graphics 1 passageway in control panel; then, choice the submenu of Graphic... which is contained the menu of Edit of the main window Image Works and open the window of Graphic Editing, choice the button of Polygon, draw the image region needed to be masked, click the Flood Fill button of the main window of Graphic Editing, click the choiced polygon region in the main window of ImageWorks, choice the submenu of Save Graphics of the main menu File, choice the Graphic 1st passageway in the save graphics dialogbox, write the passageway name in the Segment Information, click the Save\&Close button and save the masked layer.

\section{B. Modify DEM in Focus}

Open the file of DEM in Focus, choice the submenu of DEM Editing in the main menu Tools, open the two windows of Layer Selection and 2D DEM Editing, choice the DEM passageway in the windows of Layer Selection, click the button of OK; open the 1st passageway in the dialogbox of Mask Operations, choice the submenu of User Value in the main menu Area Fills Under Mask, fill in the number of elevation, click the button of Fill Pixels With Value, click the button of Interpolate in the main menu Filtering and Interpolation, open the window of save, set up the path and save the storage channel, click the button of OK and finish modifying $\mathrm{DEM}^{[7]}$.

\section{FLY! Module Features}

FLY! Module is a three-dimensional visualization software, which can run on all platforms, you can produce a variety of 3D remote sensing image. Start FLY! Module, there are two windows: FLY! And FLY! Rendering Window. FLY! Window has four main menu: File, Edit, Options, and Help; there are four tool bar: control panel, free-flying, front view and flight panel.

There are file management, displaying 3D remote sensing image, saving and exiting the module sub-single in the main menu of File. Document management has features of opening, new, importing, exporting file, adding channels, removing layers, viewing, cropping images, settings, changing projection information etc on, by means of these, users can easily manage their documents at any time.3D remote sensing image display can be divide into RGB, BW, Pseudo Color display three-dimensional display and DEM alone, what's more, transfer vector overlay in threedimensional image display. For example of Load DEM+RGB: (1)click the submenu of Load DEM + RGB in the menu of File, occur the dialog box of DEM and image; (2) click the button of Select Elevation File, occur the dialog 
box of channel selection; (3) Click the button of New File, open the file name that contains the DEM, choice the DEM channel , appear DEM channel' name in the corresponding text in the Elev, click the button of Select \& Close to open the DEM; (4) click the button of Color file, according to (2), (3) steps to choose a good RGB files; 5)click the button of Load in the dialog box of DEM and image, display threedimensional images in the FLY! Rendering Window. In addition, there is the submenu of save and display the flight path in the main menu of File.

The main menu of Edit has four sub-menus: Position, Speed, and Direction..... The submenu can adjust the designated aircraft position, altitude, flight direction and flight speed, the corresponding settings in the toolbar of the first control panel can also be achieved; the submenu of Rendering Size ... can adjust the size of three-dimensional view window; the submenu of Perspective...can set the pixel height and the magnification field of view range, viewing angle; the submenu of 3D ...can set the focal length and baseline length.

There are nine submenu in the main menu of Options, Elevation Option..., Speed Option..., Frames Per second..., Vectors..., Coordinates, Unites, Rendering Quality, Fogging Mod and Render Mode. Users can set the parameters according to their preferences.

The second toolbar is a free flight tools, press the button to start free flight 3D images, in the course of the flight the user can use the mouse in the FLY! Rendering Window to adjust the flight direction, click again the free flight instrument to stop the free flight up .

The third toolbar is front view, which can be switched between image map and DEM. The main function is designing the flight line in the light of the fourth flight panel. Design flight line as follows: choose one point in front, click the Add button in-flight panel, set up a flight; Repeat the above steps, you can easily design a good fly line, and also design the flight speed, angle of view before line is set up as needed, you can individually set the direction of the various points of view, but also can be added or deleted point and modify the flight path at any time . view flying in the flight panel view, confirm the satisfaction of using the File menu in the Save the flight path to save the path. Click Generate Move Loop, set up file formats, the number of second-zhen, file storage path and file name, adjust the size of Chen and click Generate Frames to produce a series of pictures, by the third-party software integration the films screening can be written after.

\section{APPLICATION IN ZHOUSHAN}

Zhoushan area is located in the unction of Yangtze River, Qiantang River and Yong Jiang, the main geomorphic unit is divided into two major categories of land and sea. Remote sensing images is the Spot5 one, which was filmed January 21,2005 , the range of latitude $29^{\circ} 59^{\prime} 42$ "-30 ${ }^{\circ} 19^{\prime} 57 "$, longitude $121^{\circ} 49^{\prime} 12$ "-122 ${ }^{\circ} 27^{\prime} 00^{\prime}$. Data used are 1:50000 topographic maps and 1:50000 DEM data.

\section{A. $3 D$ remote sensing image production process} Production process is shown in Figure 1.

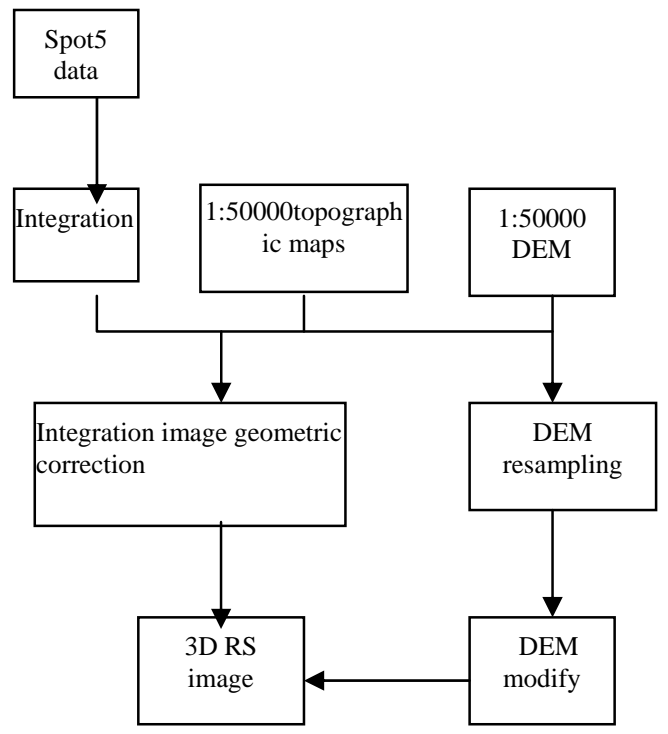

Fig. 13 D RS image production process

Used data is a Integration one ,Spot5 multispectral data and panchromatic data, we can obtain orthophoto map by Orthorectification using 1:50000 topographic maps and 1:50000 DEM in OrthoEngine in accordance with the satellite orbit model. Then we can produce 3D RS image by modifying DEM and integrating ortho image.

Generally speaking, there isn't this step which modifies DEM in 3D RS image production process, but the study area land and water are much of the possession, if we did not modify the DEM to obtain the 3D images, then the elevation displayed by DEM at the junction and the contents displayed in image do not match, some land sunkenes below the water, some water raises above the adjacent land, and the bottom elevation of all water bodies in accordance with its ups and downs do not match with the actual water height.

\section{B. Issuesing and Analysising to general practice of $3 D$ Rs image}

Zhoushan area accounted for a large part of the water body, the DEM in water body is the elevation values of the bottom, while the DEM value displayed in the image is the one of the surface water ,there will be 3 problems if we directly interge the original DEM and RS image to produce 3D RS images .

a. Seawater is above land.Fig 2(a). The elevation of Sea salt production field near the land is heigher than the one near the water .which does not meet the actual value ,even higher than the elevation of land,as seawater climbed the land.The reason for this is that the diatance of the contour of the adjacent to land is nearer than the one to sea in the interchange ShaiYan, namely, as shown in figure 2 (b),the pixel will have the elevation according to certain interpolation method during the contour between $5 \mathrm{~m}$ and $0 \mathrm{~m}$ 
when DEM is generated,the elevation of neighboring land is close to the one of land,so the elevation contours near the water will uplift in most near the middle as the boundary contour.

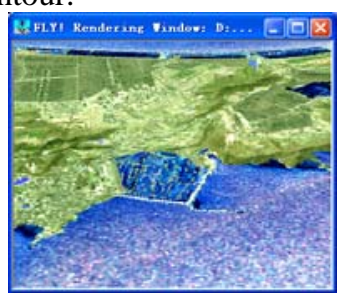

(a) raised the salt production field

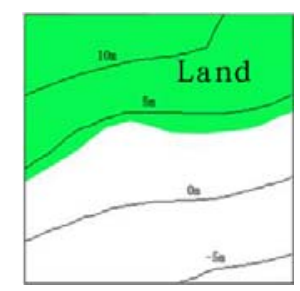

(b) analysis the contour of the salt production field
Fig. 2 examples of the sea surface above the ground instance

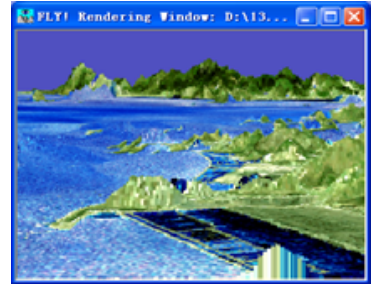

(a) depression corner of the island

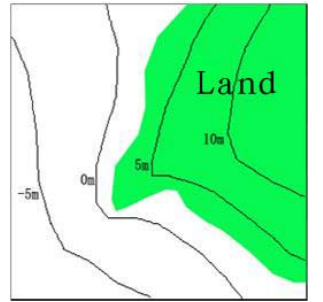

(b) island point contour analysis
Fig. 3 examples of land into the sea

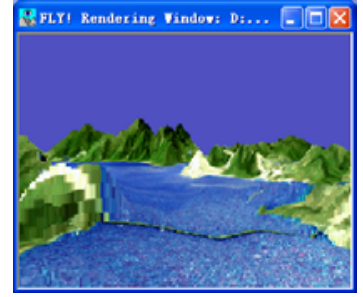

(a) zigzag bridge

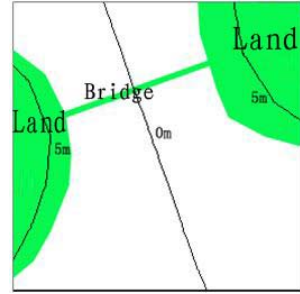

(b) contor of the bridge analysis
Fig. 4 deformation of marine structures

b. The land is under the seawater. As shown in figure 3 (a),the elevation of the island corner is below the near seawater. The reasons for this is similar to the firs one, because the distance from the island to the most nearest contour of the land is farer than of seawater,the elevation will be close to the elevation of the bottom of seawater by interpolation during DEM is generated,so the out land likes fell into the sea.

c. The building between the island deformats. As shown in figure 4 (a),the elevation of the central bridge between two islands is lower,the reason for this is that the bridge crosses several contors, as shown in figure 4 (b), the elevation of the central bridge is the one of the bottom of the seawater,and the elevation is lower,while the elevation of the ends of the bridge is the one of the land, and the elevation is higher,so the central bridge sags down.

The three problems appeared in the above images are many, just give three examples. The adopted DEM in the research area is provided according to the corresponding map of vector contou by fundamental geographic information center, so the problems in the analysis can be explained in contour.

\section{DEM modification}

For these reasons, the DEM needs to be modified so as to restore normal visual state. Modify the principle of modification is that the elevation of the seawater is to make consistent, depression of the land rose up and raised the water falling down, the Inter-island structures returne to normal form.

In order to accurately modify the DEM , the need is to put the DEM and RS images into the same file. Original DEM pixel size is $25 \mathrm{~m} \times 25 \mathrm{~m}$, RS image resolution of $2.5 \mathrm{~m}$ $\times 2.5 \mathrm{~m}$, need to resample the original DEM to make the same resolution for RS image. DEM modification is achieved primarily through the following operation.

(1) In the Focus resample the DEM, cut out the corresponding range of RS images and save it into the same pix files with the RS image;

(2) Open the pix file formed in ImageWorks, match the masked seawater area with the image map ;

(3) In Focus through DEM editing tool to modify the elevation value of the regional water- $10 \mathrm{~m}$.

\section{3Ddisplay}

Load DEM + RGB to open the modified DEM and orthophoto maps in Fly, set up various parameters such as elevation point of view, the direction of flight, line of sight direction, flight speed and height, and pixel magnification and so on, three-dimensional display such as Figure 5 (a). Figure 5 (b), (c), (d) is the with amended 3D remote sensing images corresponding to Figure 2 (a), Figure 3 (a), Figure 4 (a), we can see that the modified three-dimensional display is raised the water falling down, and depression of the land rose, and the bridge between the island is straight, which is the practical situation.

Note that the PCI of the 3D display is not very satisfactory, in the $\mathrm{x}, \mathrm{y}$ direction pixel box display was more obvious ,in the $\mathrm{z}$ direction, elevation drawing shows a clear, showing step-like. A number of parameters is more important in Fly - elevation magnification and pixel amplification, by adjusting these two parameters ,we can improve the three-dimensional display, by adjusting the smoothness and atomized we can improve the fidelity of 3D images.

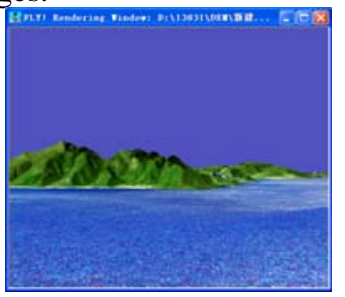

(a) 3D display

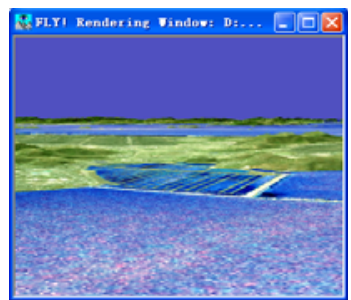

(b) Salt production field 


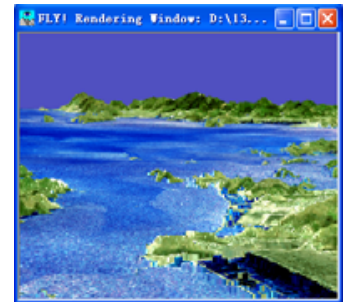

(c) corner of the island

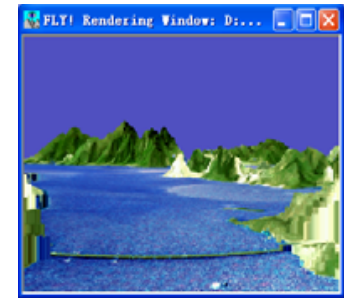

(d) brdge
Fig. 5 3D display after DEM modified

\section{CONCLUSION}

The study is to mask changes the DEM of the Zhoushan area by using the PCI software to make it how the normal surface conditions in the 3D RS images, through this study we can obtain the following conclusions: Using the PCI can accurately mask target area, quickly and easily modify the DEM value of large-scale; in three-dimensional display we can set many specific parameters to meet the different requirements of the three-dimensional display,we can easily design the flight line, three-dimensional flight; file organization systematic and manageable. The disadvantage is shown in three-dimensional effect of PCI is not too realistic, there are stretch marks.

\section{REFERENCES}

[1] Li Zhi-lin, etc.. DEM [M]. Wuhan: Wuhan University of Surveying and Mapping Press, 2000.

[2] LI Yuan-hua, JIANG Qi-gang, Zhang Bing-ren. Produce 3D RS image in large-scale by using contour data [J]. Journal of Remote Sensing, 2005, (2): $76 \sim 79$.

[3] Li Dan, Fu Zong-Tang. Discuss the three-dimensional formation [C]. ArcGIS cum sixth TECHNOLOGY ERDAS User Conference in China. Beijing: Earthquake Press. 2004.

[4] Huang Qi- huan, He Xiu-feng. Spaceborne SAR interferometry for DEM and its accuracy analysis [J]. Computer Engineering and Applications. 2008, (31) :225-227+245

[5] Dong Yue-e. Coastal terrain of the virtual environment of dynamic change [D]. East China Normal University; 2006

[6] Mei Tian-Can, Li De-ren, Qin Qian-qing. Based High Resolution Remote Sensing Image Segmentation Detection [J]. Wuhan University of Technology (Information Science). 2005, (08) :35-39

[7] PCI GIS software training tutorial [K]. October 2006. 